Ann. Zootech., I969, 18 (4), 4I9-436.

\title{
ÉTUDE DE LA DIGESTION DES FOURRAGES DANS LE RUMEN PAR LA MÉTHODE DES SACHETS DE NYLON; LIAISONS AVEC LA VALEUR ALIMENTAIRE
}

\author{
C. DEMARQUiLlY et M. CHENOST
}

avec la collaboration technique de Jacqueline Aubry, Danièle Chevalier et Marie Jailler

Station de Recherches sur l'Elevage des Ruminants, Centre de Recherches de Clermont-Ferrand, 63-Saint-Genès-Champanelle

Institut national de la Recherche agronomique

\section{SOMMAIRE}

La méthode des sachets de nylon permet de suivre la digestion des fourrages ou des organes des plantes fourragères dans le rumen. Celle-ci est pratiquement terminée au bout de 48 heures de séjour dans le rumen. Nous appelons digestibilité I 2,24 ou 48 heures en sachets, le pourcentage de matière sèche disparue des sachets après I 2,24 ou 48 heures de séjour dans le rumen. $\mathrm{La}$ digestibilité 48 heures des fourrages est liée essentiellement à la digestibilité in vivo et permet donc de la prévoir.

La digestibilité $\mathbf{I} 2$ ou 24 heures est liée à la fois à la digestibilité in vivo et à la nature botanique du fourrage. La luzerne est digérée plus rapidement que les graminées et le Ray-grass d’Italie plus rapidement que la Fétuque élevée.

La digestibilité $\mathrm{I} 2$ heures permet de prévoir les quantités de fourrages verts ou d'ensilages ingérées; la digestibilité 24 heures permet de prévoir les quantités de foin ingérées. Le produit des deux digestibilité I 2 (ou 24 heures) et 48 heures permet de prévoir la quantité de matière organique digestible ingérée qui caractérise au mieux la valeur alimentaire des fourrages. La précision de la prévision est en moyenne aussi bonne que celle obtenue à partir de la digestibilité in vivo et est bien meilleure que celle obtenue à partir de la teneur en cellulose brute des fourrages.

\section{INTRODUCTION}

Ia valeur alimentaire d'un fourrage dépend de deux facteurs d'égale importance : sa valeur nutritive, plus spécialement sa valeur énergétique dont le meilleur critère est le coefficient de digestibilité de la matière organique, et son acceptabilité

Annales de Zootechnie. - rg69. 
ou quantité de matière sèche volontairement ingérée par un animal à qui on offre ce fourrage à volonté.

La mesure de la digestibilité des fourrages et sa prévision ont fait l'objet de très nombreux travaux tant en France qu'à l'étranger. On a proposé depuis fort longtemps des méthodes chimiques clont aucune ne donne entièrement satisfaction. De nombreux auteurs se sont donc orientés depuis une dizaine d'années vers les méthodes biologiques utilisant le jus de rumen d'animaux fistulisés (cf. revue de Jonsson, I 966) car elles donnent une estimation plus précise de la cligestibilité que les méthodes chimiques de rapidité comparable.

İn revanche, on a pendant très longtemps négligé de mesurer les quantités ingérées ad libitum, et à plus forte raison on ne s'est pas intéressé à les prévoir. I.es facteurs de variation de l'acceptabilité des fourrages sont done mal connus. On a longtemps attribué ces variations de l'acceptabilité des fourrages verts à celles de leurs qualités gustatives pour l'animal qui les reçoit, d'où les nombreux essais de "palatabilité » effectués (cf. revue de Iviss, i955). On sait maintenant que la quantité des fourrages secs ingérée par le ruminant est avant tout liée à la distension des réservoirs gastriques et notamment du rumen (cf. revue de BAI,CH et CAMli,Is, I 962), et qu'elle présente en particulier une liaison positive étroite avec la digestibilité du fourrage (BIAXTER et al., Ig6I). De même que CoRIB:TT et al. (Ig63), nous avons

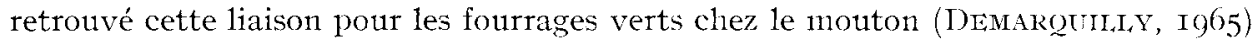
et che\% la vache (I) MARQUi, I, I966). Nous avons cependant montré (ule la liaison entre la quantité de matière sèche ingérée et la digestibilité n'était vraiment étroite qu'à l'intérieur du même cycle de végétation de la même espèce. Il en résulte que des fourrages de même digestibilité mais de familles, d'espèces ou de cycles végétatifs différents peuvent être ingérés en quantité très différente ; en particulier, les Légumineuses sont ingérées en plus grande quantité que les Graminées et les repousses en plus grande quantité que les premiers cycles végétatifs. I)ans les mêmes conditions, au contraire, les quantités de cellulose brute digestible ingérées sont sensiblement les mêmes. Pour expliquer ce fait, nous avions supposé que c'était plus la vitesse cle digestion que la digestibilité des fourrages qui était le facteur essentiel de leur acceptabilité. Nous ne faisions en fait que reprendre 1'hypothèse émise par CrAmptoN (I957). DONEFER et al. (I960) ont d'ailleurs mis en évidence une liaison entre la quantité de matière sèche ingérée et la quantité de cellulose dégradée après un temps court (I2 heures) de fermentation in vitro.

Depuis plusieurs années nous poursuivons à la Station de Recherches sur l'Élevage des Ruminants l'étude systématique de la mesure et de la prévision de la valeur alimentaire des fourrages verts et conservés. Dans le cadre de cette étude nous avons étudié s'il était possible d'utiliser la méthode des sachets de nylon proposée par VAN KEUREN et Heinemans (I962), méthode qui est dérivée de celle des poches de soie de Quin et al. (I938), pour :

- d'une part, suivre la digestion des fourrages ou des organes végétatifs de plantes fourragères dans le rumen;

- d'autre part, relier les quantités digérées dans les sachets après différents temps de séjour dans le rumen à la digestibilité et à l'acceptabilité des fourrages. 


\section{MATÉRIEL ET MÉTHODES}

\section{Fourrages}

Nous avons utilisé 62 fourrages verts, 58 foins et 77 ensilages. Ces fourrages ont été choisis de familles botaniques, d'espèces et de cycles végétatifs différents, parmi ceux dont nous avons étudié de I965 à I 967 la cligestibilité et l'acceptabilité sur des moutons suivant les méthodes que nous avons précédemment décrites (DemarQUilly et JARRIgE, I964; DemarQUilly, I965).

Nous avons d'abord étudié la cinétique de la digestion des feuilles et des tiges de deux luzernes et de trois graminées dont l'évolution avec l'âge de la digestibilité et de l'acceptabilité était mesurée sur des moutons. Les feuilles et les tiges de ces plantes ont été séparées, sćchées à l'étuve à $80^{\prime \prime} \mathrm{C}$, puis très grossièrement broyées dans un cutex. Nous avons ainsi étudié :

- en I964 (cssai I) des feuilles et (les tiges de Luzerne prélevées toutes les deux semaines au cours du I $^{\text {er }}$ cycle végétatif ( 4 prélèvements) et au début et à la fin (les $2^{\mathrm{e}}, 3^{\mathrm{e}}$ et $4^{\mathrm{e}}$ cycles végétatifs:

- en I965 (cssai 2) cles feuilles et des tiges de Luzerne, de Ray-grass d'Italie, cle Fétuque élevée et de Dactyle, prélevées toutes les deux semaines au cours du I er cycle végétatif.

Nous avons ensuite mesuré l'importance de la digestion au bout de $12-24-48$ et 72 heures des 20 échantillons de plante entière correspondant aux feuilles et aux tiges étudiées dans l'essai 2 , et (Ie $3+$ foins (essai 3) pour étudier qu'elles étaient les clurées de la digestion qui permettaient de prévoir au mieux la digestibilité et l'acceptabilité de ces fourrages. Ces durécs ayant été déterminées nous avons alors mesuré l'importance de la digestion au bout de 12 ou 24 heures et 48 heures de +3 fourrages verts, 20 foins et i 7 ensilages (essai 4 ) pour la relier à la valeur alimentaire de ces fourrages. 'Tous les fourrages étudiés dans les essais 3 et + étaient des échantillons représentatifs des fourrages offerts penclant les périodes de mesure de la digestibilité et de la quantité ingérée. Ils avaient été séchés pendant 24 heures à l'étuve à $80^{\circ} \mathrm{C}$ avant d'être finement broyés dans un broyeur muni d'une grille à maille de $0,8 \mathrm{~mm}$ de diamètre. Les valeurs moyennes et extrêtmes de Ia cligestibilité et de l'acceptabilité des échantillons utilisés lors cles différents essais sont présentées dans le tableau $x$.

TABLEAU I

Digestibilité et acceptabilité des différents échantillons de fourrages étudiés au cours des différents essais

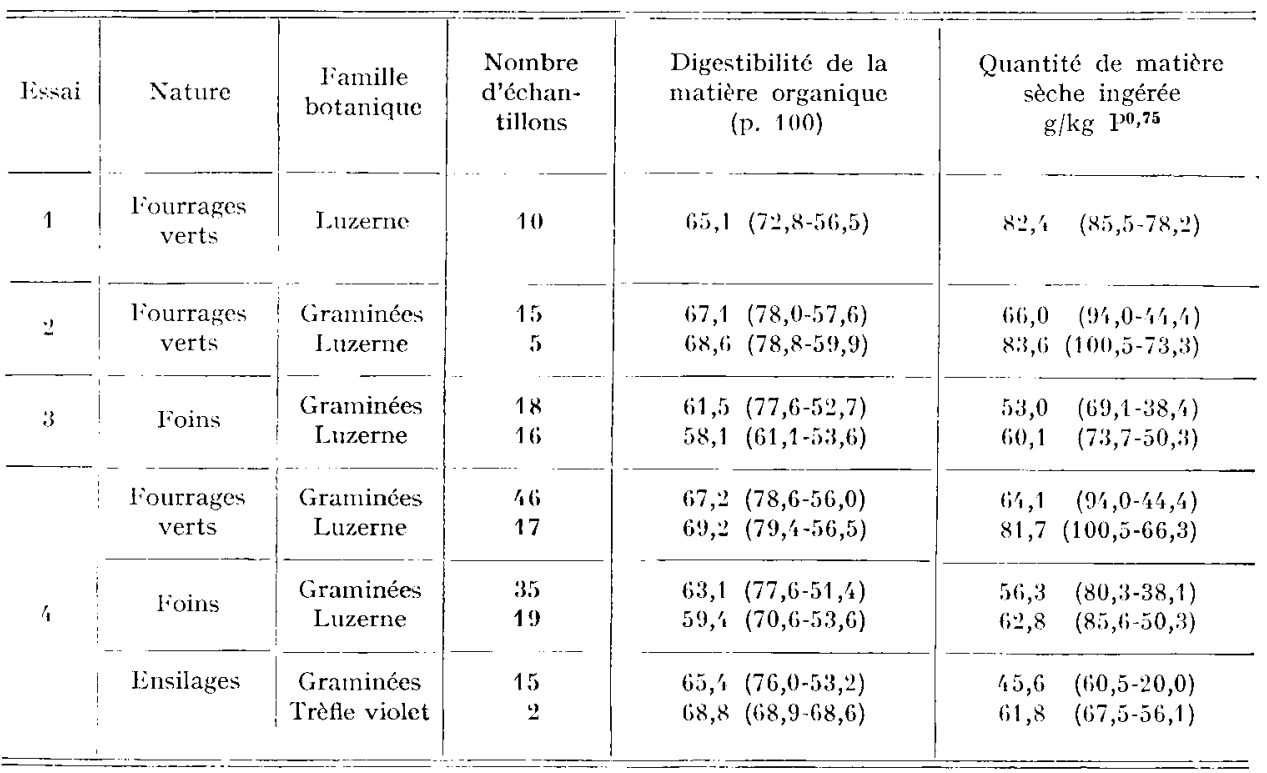




\section{Technique des sachets de nylon}

Environ $3 \mathrm{~g}$ de matière sèche de fourrage broyé sont pesés et introduits dans un sachet de tissu de nylon à mailles fines $(\leqslant 50 \mu)$. Ce sachet, qui mesure environ $5 \mathrm{~cm} \times 7 \mathrm{~cm}$, est fabriqué sans couture mais avec des soudures à chaud. Une fois l'échantillon introduit, le dernier côté est fermé par deux soudures parallèles entre lesquelles on fixe un nillet permettant de suspendre le sachet. Les sachets sont mis 24 heures à l'étuve avant d'être pesés. Ils sont alors attachés par un fil de nylon à un anneau de plomb de I kg enrobé de matière plastique qui est déposé dans le sac ventral clu rumen d'une vache portcuse d'une large canule du rumen. Nous avons introduit au maximum $4^{\circ}$ sachets à la fois et utilisé 3 sachets par fourrage et par temps de séjour. Les sachets ont été retirés du rumen après respectivement 24,48 et 72 heures dans l'essai I et I $2,24,48$ et 72 heures dans les essais 2 et 3 , I 2 ou 24 et 48 heures dans l'essai 4 . Dès leur sortie du rumen ils sont abondamment lavés puis séjournent 48 heures dans une solution acide de pepsine identique à celle utilisée par 'lilley et Terky (I963). Ils sont alors de nouveau lavés, séchés et pesés. Le pourcentage de matière sèche disparu est appelé digestibilité $12,24,48$ ou 72 heures en sachets.

Dans les essais I et 2 chaque échantillon de fourrage a été étudié simultanément sur dcux vaches qui recevaient 4 repas $\left(6 \mathrm{~h}, 9 \mathrm{~h}, \mathrm{I}_{4} \mathrm{~h}\right.$ et $\left.\mathrm{I} 8 \mathrm{~h}\right)$ de luzerne verte fauchée chaque matin. A partir de l'essai 3 nous n'avons utilisé qu'une seule vache recevant un bon foin de luzerne en deux repas par jour, à 6 et 18 heures. Les sachets ont été, dans chaque essai, déposés dans le rumen à 9 heures. Au cours de l'essai 3 nous nous sommes progressivement aperçus qu'il était nécessaire d'incorporer dans chaque série un foin standard et cela pour deux raisons ; d'une part, l'activité cellulolytique du jus de rumen peut éventuellement varicr un peu d'un jour à l'autre, même quand la vache reçoit toujours le même foin; ces variations d'activité cellulolytique ont une influence parfois non négligeable sur les valeurs obtenues à I 2 heures. D'autre part, les valeurs obtenues pour des fourrages broyés finement (et c'est le cas pour les essais 3 et 4) peuvent dépendre dans une certaine mesure du tissu de nylon employé. Or, il est très difficile de retrouver un tissu ayant exactement les mêmes caractéristiques d'un achat à l'autre.

Dans l'essai 4 les valeurs obtenues à chaque série ont donc été réajustées en les multipliant par le rapport :

\section{Moyenne pour l'ensemble des séries des valeurs du foin standard \\ Valeur du foin standard pour la série considérée}

\section{RÉSULTATS}

\section{Adaptation de la méthode}

Nous avons d'abord étudié si les valeurs obtenues dépendaient de l'animal utilisé et quel était le nombre de sachets nécessaire pour obtenir une précision satisfaisante. Pour cela nous avons mesuré la digestion 24,48 et 72 heures dans le rumen de 2 vaches, du papier-filtre et de 4 échantillons de feuilles et de tiges de luzerne, prélevés à intervalles de 2 semaines au cours du premier cycle de végétation. Il y a eu 4 séries de mesures, une par prélèvement, chaque échantillon étant étudié en triple ( $3 \mathrm{~g}$ de matière sèche) pour chaque temps de séjour dans le rumen et pour chaque vache. A leur sortie du rumen, les sachets ont été lavés soigneusement à l'eau, séchés puis pesés.

La méthode des sachets de nylon permet de suivre la digestion des fourrages ou du papier filtre dans le rumen (fig. I). Cependant, pour les substrats se dégradant rapidement (feuilles), la valeur 72 heures de la digestibilité est plus faible que la valeur 48 heures. Cela nous semblait résulter du fait que les sachets étaient d'autant plus difficiles à laver qu'ils avaient séjourné plus longtemps dans le rumen. Les sachets ayant été conservés, nous les avons repris pour les soumettre à une digestion pepsique en les laissant tremper 48 heures dans une solution acide de pepsine main- 
tenue à $40^{\circ}$, par analogie avec ce qui se fait dans la méthode de digestibilité in vitro de TILLEY et al. (I960). Ce traitement augmente les digestibilités 24,48 et 72 heures en moyenne de $2,5-3$ points, $3-4$ points et $7-8$ points respectivement. L'augmentation est indépendante du substrat considéré (fourrage ou papier-filtre), ce qui permet de penser que le traitement agit surtout en permettant un lavage correct des sachets. Il élimine la diminution apparente de la digestibilité d'un substrat dont la digestion est terminée (fig. I). Nous l'avons adopté systématiquement pour les études ultérieures, et les sachets après avoir été lavés à leur sortie du rumen séjournent 48 heures dans une solution acide de pepsine avant d'être séchés et pesés.

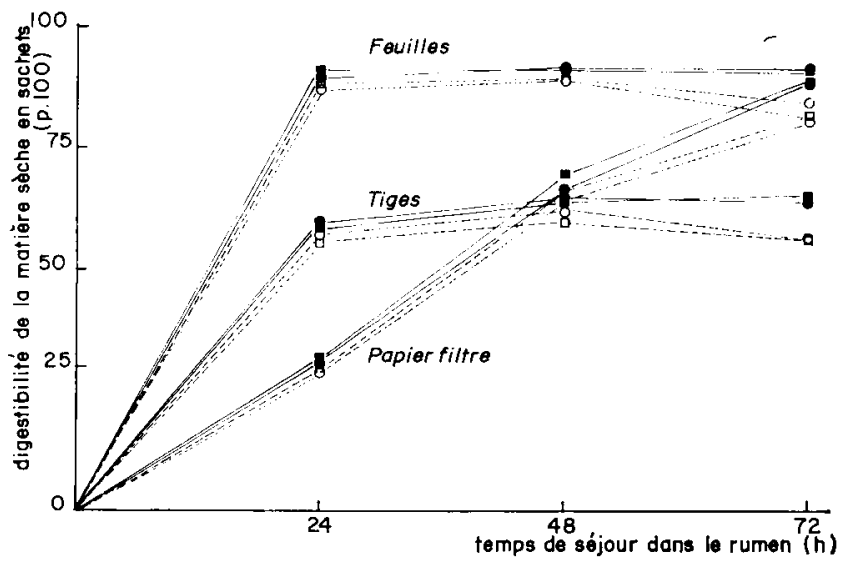

FIG. I. - Cinétique de la digestion dans le rumen du papier-filtre et des feuilles et des tiges de luzerne mesurée chez 2 taches par la technique des sachets de nylon sans et après avoir soumis les sachets à une digestion pepsique à leur sortie du rumen.

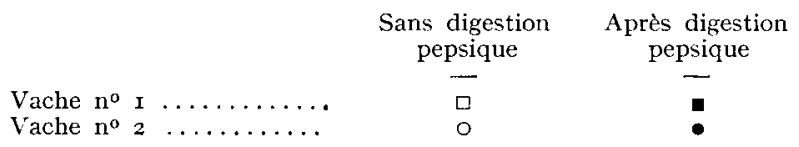

Les valeurs obtenues diffèrent très peu entre les deux vaches (fig. I) : en moyenne pour les 4 séries de $0, I_{5}$ point pour les feuilles, $I, 45$ point pour les tiges, I,o point pour le papier-filtre. Ces différences ne sont pas significatives mais peuvent le devenir pour une série donnée; c'est ainsi que, lors de la $3^{\mathrm{e}}$ série de mesures elles ont atteint 3,0 points en moyenne; elles étaient d'autant plus faibles que le temps de séjour dans le rumen était important: 6,5 points à 24 heures, I,O point à 48 heures et 0,2 point à 72 heures pour les substrats luzerne. Il est donc indispensable de faire les mesures simultanément sur deux vaches ou de placer des fourrages standards dans chaque série.

Une étude statistique des résultats des 4 séries de mesure indique que le nombre de sachets nécessaires pour avoir une précision d'estimation de $\mathrm{r}, 2$ et $3 \mathrm{p}$. Ioo est respectivement le suivant: 8,2 et I sachets pour les feuilles, I4,4 et 2 sachets pour les tiges.

Pour les mesures ultérieures nous avons donc adopté 3 sachets par temps de séjour pour les substrats fourrages, et abandonné le substrat papier-filtre à cause de la variabilité des résultats ; il faudrait Io sachets pour obtenir une précision de $5 \mathrm{p}$. Ioo au temps de séjour 48 heures. 


\section{Cinétique de la digestion dans le rumen des feuilles, des tiges et des plantes entières}

Les résultats des mesures effectuées sur les 4 cycles de Luzerne en I964, et sur les premiers cycles de Ray-grass d'Italie, de Fétuque élevée, de Dactyle et de Luzerne en 1965 sont présentés dans les figures 2 et 3 .

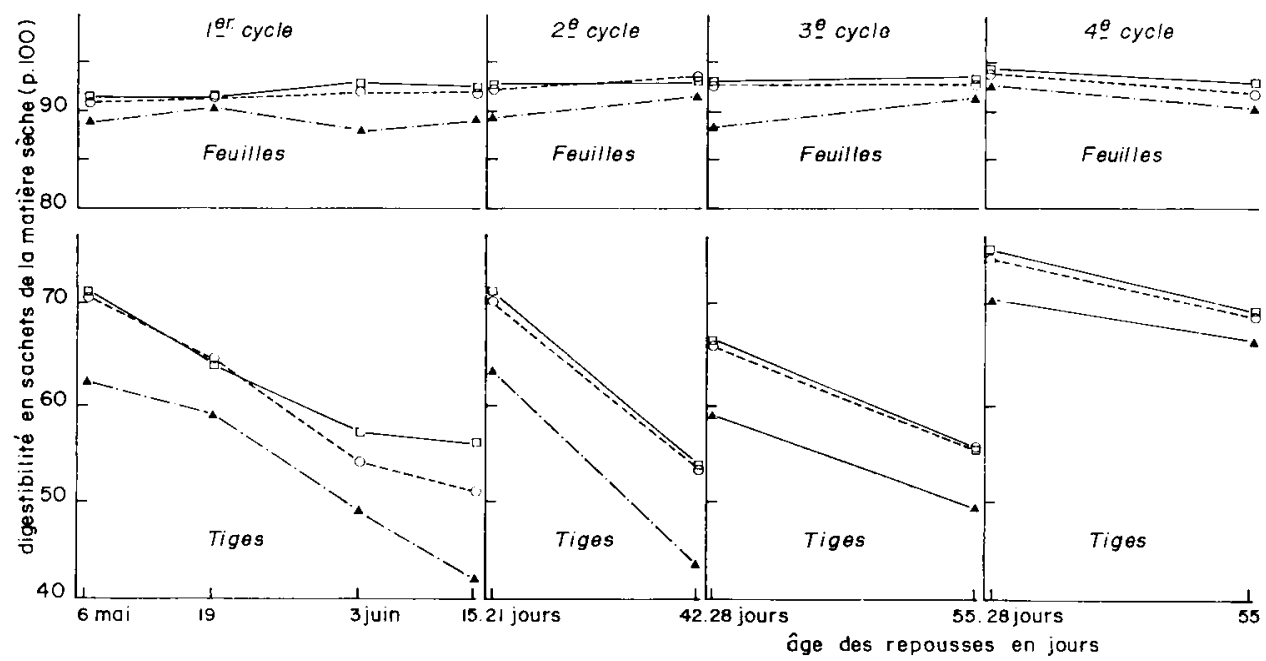

FIG. 2. - Évolution azec l'âge au cours des cycles successifs de la digestibilité en sachets $\dot{a} 24,48$ et 72 heures des fenilles et des tiges de luzerne

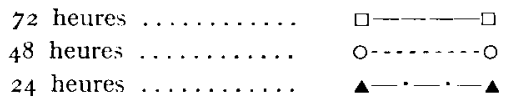

La digestion des feuilles de luzerne est pratiquement terminée au bout de 24 heures de séjour dans le rumen, et les valeurs obtenues à 48 ou $7^{2}$ heures sont voisines de 92-93 p. Ioo, quels que soient l'âge de la plante et le numéro du cycle de végétation. Fn revanche, la digestion des limbes de graminées est plus lente et elle demeure importante entre 24 heures et 48 heures de séjour (temps au bout duquel la digestion est alors presque terminée), notamment pour la Fétuque élevée. Son importance dépend du stade de végétation de la plante; aussi les valeurs obtenues après 48 heures de séjour dans le rumen sont élevées au début du I er cycle $(82,5$ à 92,5 suivant les espèces) puis elles diminuent assez régulièrement avec 1'âge d'environ $0,17,0,20$ et 0,30 point par jour, respectivement pour le Ray-grass, la Fétuque et le Dactyle.

La digestion des tiges de Luzerne est plus lente que celle des feuilles. Elle n'est pratiquement achevée, comme celle des tiges de graminées d'ailleurs, qu'après 48 heures de séjour dans le rumen. La digestibilité 48 heures des tiges de Luzerne est toujours nettement inférieure à celle des feuilles, et elle diminue avec l'âge de la plante. La diminution est variable suivant le numéro du cycle de végétation : $0,63,0,95,0,34$ et 0,17 point par jour respectivement aux I ${ }^{\mathrm{er}}, 2^{\mathrm{e}}, 3^{\mathrm{e}}$ et $4^{\mathrm{e}}$ cycles. La $^{\mathrm{C}}$ digestibilité 48 heures des tiges de graminées peut être aussi élevée que celle des limbes au début du premier cycle de végétation, mais elle diminue beaucoup plus 


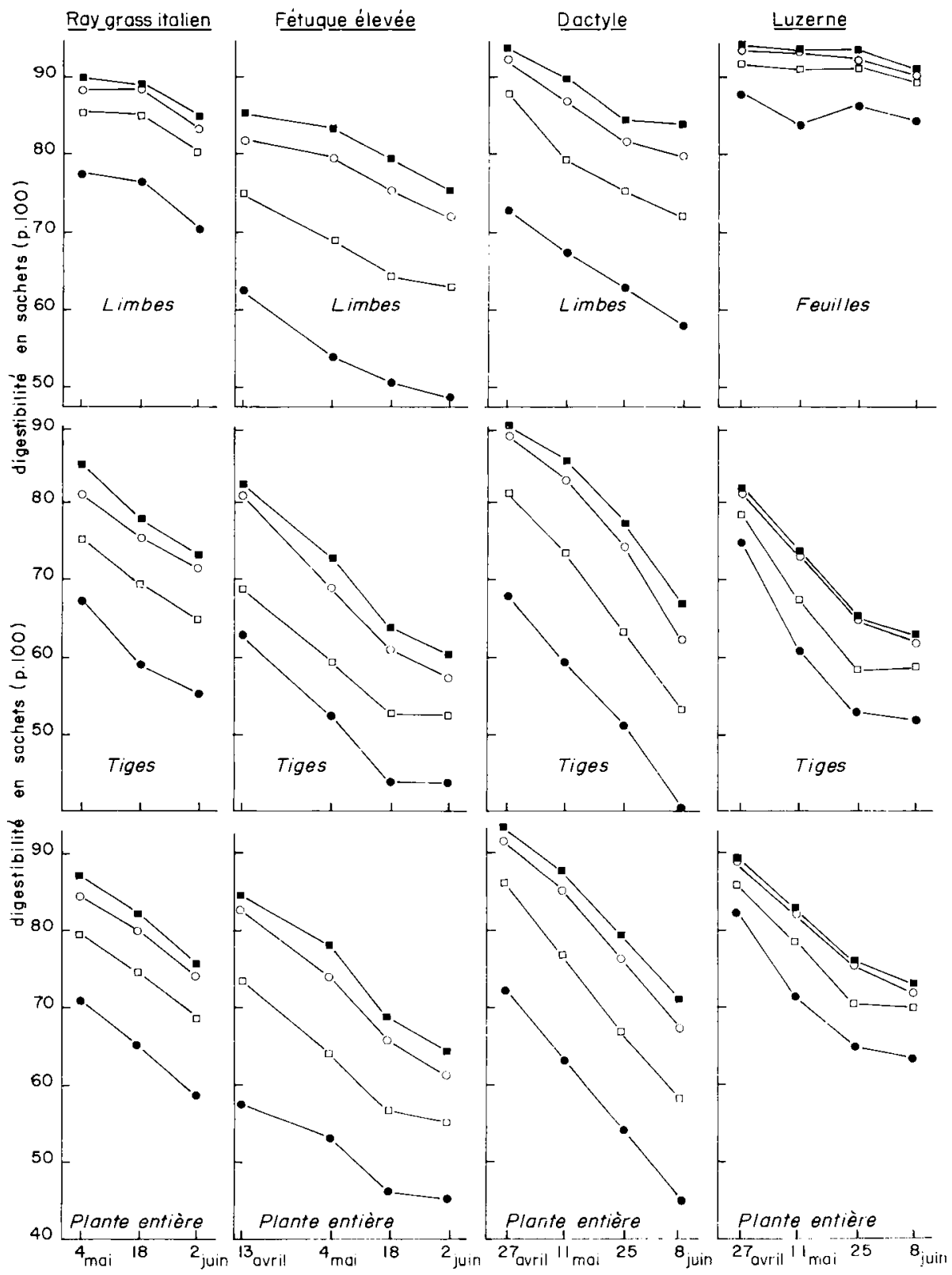

FIG. 3. - Évolution au cours du premier cycle de végétation de la digestibilité en sachets à 12, 21, 48 et 72 heures des feuilles, des tiges et des plantes entières de Ray-grass d'Italie, de Fétuque élevéc, de Dactyle et de Luzerne.
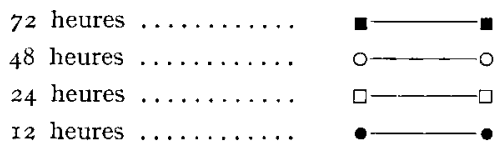
vite avec l'âge : respectivement $0,33,0,47$ et 0,75 point par jour, pour le Ray-grass, la Fétuque et le Dactyle.

Les digestibilités $12,24,48$ et 72 heures de la plante entière vont donc diminuer avec l'âge de la plante (fig. 3), d'une part parce que la proportion de feuilles diminue au bénéfice de la proportion de tiges, d'autre part parce que les digestibilités propres des limbes et des tiges diminuent.

Il est intéressant de constater que des fourrages de même digestibilité in vivo ont des digestibilité 48 ou 72 heures pratiquement identiques (ces valeurs doivent donc être en liaison étroite avec la digestibilité in vivo des fourrages), mais des digestibilités 12 ou 24 heures très différentes (fig. 4). La Luzerne est digérée plus vite que

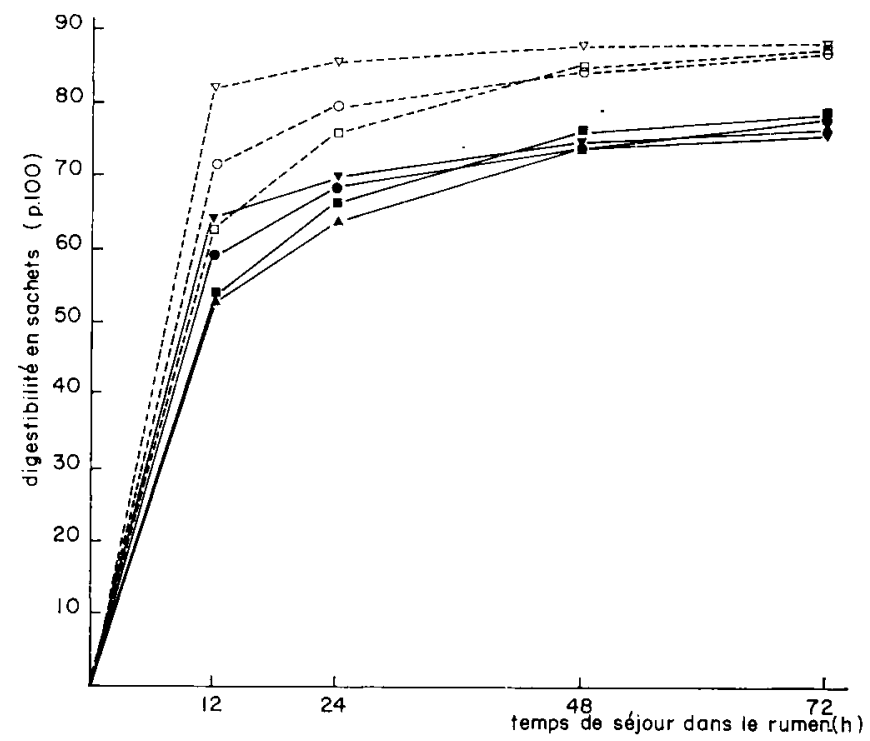

FIG. 4. - Cinétique de la digestion dans le rumen de fourrages de même digestibilité

\begin{tabular}{|c|c|c|}
\hline$\nabla \cdots \cdots$ & Luzerne & dig. mo: 78,8 \\
\hline $0 \cdots \cdots$ & Ray-grass & dig. mo : 76,8 \\
\hline$\square \cdots \cdots$ & 1)actyle & dig. mo : 75, I \\
\hline$\nabla-$ & Luzerne & dig. noo: 66,9 \\
\hline$\bullet-$ & Ray-grass & dig. mo \\
\hline & Dactyle & dig. nno \\
\hline$-\boldsymbol{A}$ & l'étuque & ig. mo \\
\hline
\end{tabular}

le Ray-grass d'Italie et ce dernier plus vite que le Dactyle et la Fétuque élevée. Cependant la digestibilité I2 heures ne peut servir à estimer la vitesse de digestion des fourrages que si ceux-ci ont la même digestibilite 48 heures. En effet elle dépend à la fois de la plante considérée et de la digestibilité 48 hetures (fig. 5) c'est-à-dire de la digestibilité $i n$ vivo. Aussi proposons-nous d'appeler vitesse de digestion le rapport digestibilité I2 heures digestibilité 48 heures $\times$ roo. Les valeurs moyennes et extrêmes obtenues au début et à la fin du premier cycle de végétation sont présentées dans le tableau 2. Elles confirment que la L,uzerne est digérée plus rapidement que les graminées et le Raygrass italien plus rapidement que le Dactyle et la Fétuque élevée. Elles montrent en outre que la vitesse de digestion des feuilles est plus élevée que celle des tiges sauf 
pour la Fétuque élevée et qu'elle diminue beaucoup moins vite avec l'âge de la plante. Ces différences de vitesse de digestion entre organes pour une plante donnée sont

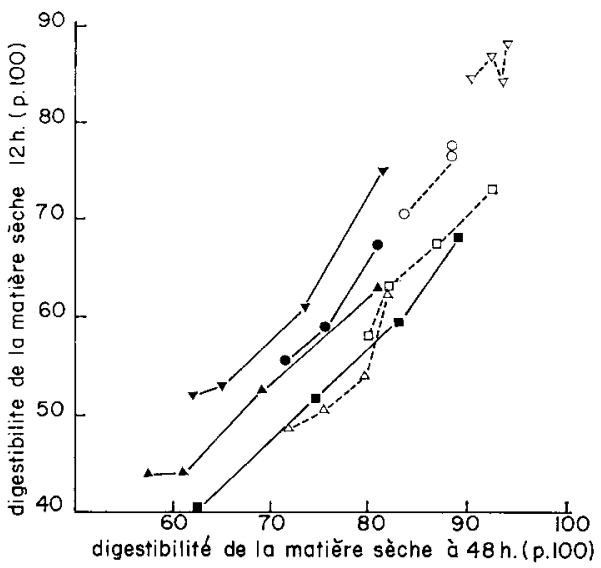

FIG. 5. - Liaisons entre la digestibilité 12 heures et la digestibilité 48 heures des feuilles et des tiges de Ray-grass, de Dactyle, de Fétuque élevée et de Luzerne

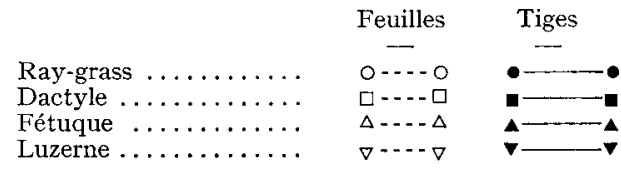

TABLEAU 2

Valeurs moyennes et valeurs extrêmes (en début et fin de cycle) de la vitesse de digestion dans le rumen des feuilles,

des tiges et des plantes entières, mesurée par le rapport $\frac{\text { Digestibilité } 12 \text { heures }}{\text { Digestibilité } 48 \text { heures }} \times 100$

\begin{tabular}{c|c|c|c}
\hline \hline Nature du fourrage & $\begin{array}{c}\text { Feuilles } \\
\text { ou limbes }\end{array}$ & $\begin{array}{c}\text { Tiges } \\
\text { ou } \\
\text { tiges + gaines }\end{array}$ & Plante entière \\
\hline Dactyle & $\begin{array}{c}76,5 \\
(79,0 \text { à } 72,5)\end{array}$ & $\begin{array}{c}70,5 \\
(77,5 \text { à } 64,0)\end{array}$ & $\begin{array}{c}72,0 \\
(78,5 \text { à } 66,0)\end{array}$ \\
\hline Fétuque élevée & $\begin{array}{c}67,0 \\
(67,5 \text { à } 67,0)\end{array}$ & $\begin{array}{c}75,0 \\
(76,0 \text { à } 72,5)\end{array}$ & $\begin{array}{c}72,5 \\
(74,5 \text { à } 71,0)\end{array}$ \\
\hline Ray-grass italien & $\begin{array}{c}86,0 \\
(87,5 \text { à } 84,5)\end{array}$ & $\begin{array}{c}89,5 \\
(83,5 \text { à } 77,5)\end{array}$ & $\begin{array}{c}85,5 \\
(85,0 \text { à } 79,0)\end{array}$ \\
\hline Luzerne & $\begin{array}{c}92,5 \\
(93,5 \text { à } 90,0)\end{array}$ & $\begin{array}{c}82,0 \\
(92,0 \text { à } 80,5)\end{array}$ & $\begin{array}{c}88,0 \\
(93,0 \text { à } 85,5)\end{array}$ \\
\hline \hline
\end{tabular}

dues en partie au fait que la digestibilité 48 heures des feuilles est plus élevée et diminue beatcoup moins vite que la digestibilité 48 heures des tiges. 


\section{Relations avec la valeur alimentaire des fourrages}

Dans une première étape nous avons cherché à déterminer la durée (I2, 24 ou 48 heures) de la digestion qui permettait de prévoir au mieux la digestibilité et 1'acceptabilité des fourrages mesurées sur des moutons. Cette étude a été faite avec 20 échantillons de fourrages verts (dont I5 sont ceux dont nous avions mesuré la cinétique de la digestion des feuilles et des tiges) et 34 échantillons de foins : 18 foins de graminées (7 Fétuque élevée, 4 Ray-grass d'Italie, 2 Dactyle, 5 prairies permanentes) et 16 foins de luzerne.

La prévision de la digestibilité in vivo des fourrages verts s'améliore avec la durée de la digestion en sachets et celle de la quantité ingérée devient moins bonne (tab1. 3). La digestibilité in vivo peut être prévue de façon très satisfaisante à partir de la digestibilité 48 heures $(r=0,895)$ et la quantité ingérée à partir de la digestibilité I2 heures $(r=0,944)$ (tab1. 3, fig. 6). Il n'y a aucun avantage à considérer la digestibilité de la matière sèche insoluble dans l'eau (qui est un critère approché des constituants membranaires du fourrage) plutôt que la digestibilité de la matière sèche (tabl. 3).

\section{TABLEAU 3}

Coefficients de corrélation et écarts-type réduits entre la digestibilité et l'acceptabilité des fourrages verts et la digestibilité en sachets à 12,24 et 48 heures

\begin{tabular}{|c|c|c|c|c|c|c|c|c|c|}
\hline & \multirow{2}{*}{$\begin{array}{l}\text { Nbre } \\
\text { échan- } \\
\text { tillons }\end{array}$} & & \multirow{2}{*}{$\begin{array}{l}\text { Digesti- } \\
\text { bilité } \\
\text { in vivo }\end{array}$} & \multicolumn{3}{|c|}{$\begin{array}{l}\text { Digestibilité de la matière } \\
\text { sèche en sachets }\end{array}$} & \multicolumn{3}{|c|}{$\begin{array}{l}\text { Digestibilité de la matière } \\
\text { sèche non extractible à l'eau }\end{array}$} \\
\hline & & & & 12 heures & 2't heures & 48 heures & 12 heures & 24 heures & 48 heures \\
\hline $\begin{array}{l}\text { Digestibilité in vivo } \\
\text { de la matière } \\
\text { organique (p. 100) }\end{array}$ & 20 & $\begin{array}{c}r \\
\text { Syx }\end{array}$ & & $\begin{aligned} & 0,748 \\
+ & 4,59\end{aligned}$ & $\begin{aligned} & 0,811 \\
\pm & 3,74\end{aligned}$ & $\begin{aligned} & 0,895 \\
\pm & 3,08\end{aligned}$ & $\begin{array}{ll} & 0,695 \\
\pm & 4,97\end{array}$ & $\begin{aligned} & 0,807 \\
\pm & 4,08\end{aligned}$ & $\begin{aligned} & 0,849 \\
\pm & 3,65\end{aligned}$ \\
\hline $\begin{array}{c}\text { Quantité de matière } \\
\text { sèche ingérée } \\
\left(\mathrm{g} / \mathrm{kg} \mathrm{P}^{\mathbf{0 , 7 5}}\right)\end{array}$ & 20 & $\begin{array}{c}\gamma \\
\text { Syx }\end{array}$ & $\begin{aligned} & 0,814 \\
-1- & 8,18\end{aligned}$ & $\begin{aligned} & 0,914 \\
\pm & 4,82\end{aligned}$ & $\begin{aligned} & 0,897 \\
\perp & 6,44\end{aligned}$ & $\begin{aligned} & 0,823 \\
+ & 8,30\end{aligned}$ & $\begin{aligned} & 0,941 \\
+ & 4,93\end{aligned}$ & $\begin{aligned} & 0,878 \\
\pm & 6,98\end{aligned}$ & $\begin{aligned} & 0,791 \\
\pm & 8,94\end{aligned}$ \\
\hline
\end{tabular}

La digestibilité in vivo des foins, notamment ceux de graminées, peut être estimée de façon très satisfaisante à partir de la digestibilité en sachets à 48 heures (tab1. 4). Le coefficient de corrélation obtenu pour les foins de légumineuses n'est pas significatif, mais cela s'explique par le fait que les digestibilités in vivo de ces foins étaient très peu variables : $58, \mathrm{I} \pm 2,5$. Quand on considère l'ensemble des foins, ce sont les digestibilités à I 2 ou 24 heures qui permettent la meilleure estimation des quantités ingérées ; les droites de régression sont d'ailleurs pratiquement confondues pour les graminées et les légumineuses.

Compte tenu de ces résultats, nous avons alors mesuré la digestibilité en sachets de 42 fourrages verts, 24 foins et $I 7$ ensilages qui sont venus s'ajouter aux 20 fourrages verts et aux 34 foins déjà étudiés. Pour ces nouveaux échantillons nous nous sommes cependant limités aux temps de séjour suivants dans le rumen : I2 et 48 heures pour 

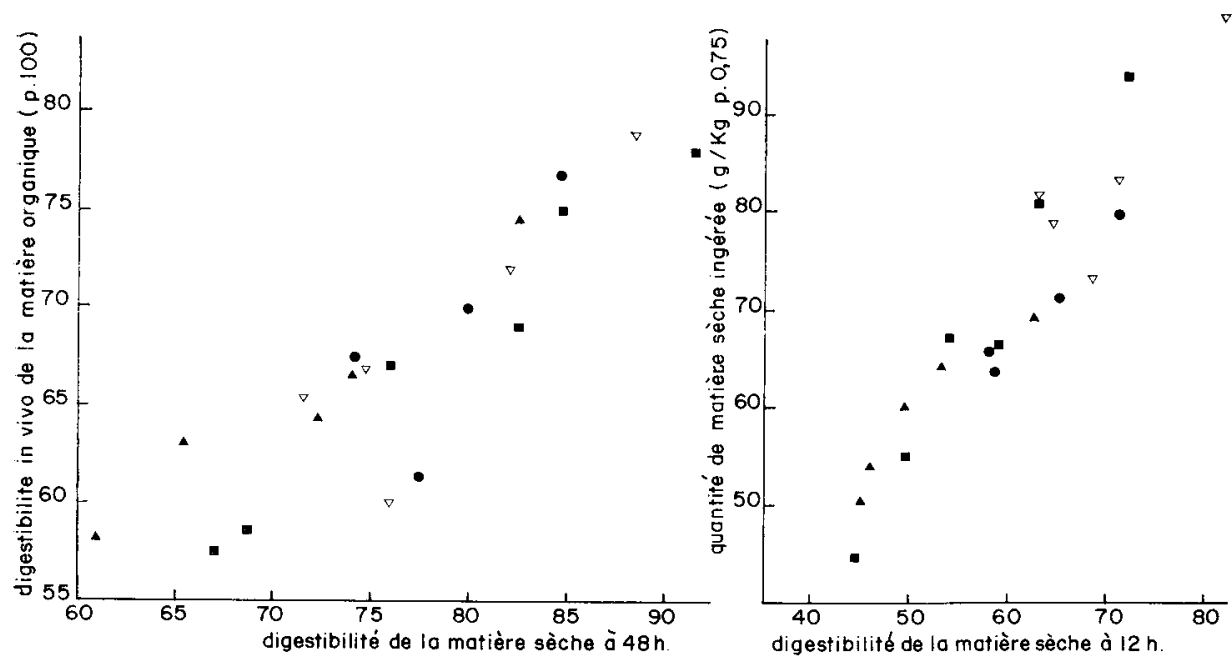

FIG. 6. - Liaisons entre la digestibilité in vivo et la digestibilité 48 heures et entre la quantité de matière sèche ingérée et la digestibilité 12 houres pour des fourrages verts

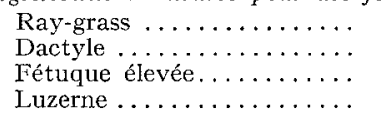

TABLEAU 4

Coefficients de corrélation et écarts-type réduits entre la digestibilité et l'acceptabilité des foins et la digestibilité en sachets à 12, 24 et 48 heures

\begin{tabular}{|c|c|c|c|c|c|c|c|}
\hline & & Nombre & & Digesti- & \multicolumn{3}{|c|}{ Digestibilité de la matière sèche à } \\
\hline & & tillons & & in vivo & 12 heures & 24 heures & 48 heures \\
\hline \multirow{3}{*}{$\begin{array}{l}\text { Digestibilité in vivo } \\
\text { de la matière } \\
\text { organique (p. 100) }\end{array}$} & Luzerne & 16 & $\begin{array}{c}r \\
\text { Syx }\end{array}$ & & $\begin{aligned} & 0,207 \mathrm{NS} \\
+ & 2,51\end{aligned}$ & $\begin{aligned} & 0,318 \mathrm{NS} \\
\pm & 2,44\end{aligned}$ & $\begin{aligned} & 0,247 \mathrm{NS} \\
+ & 2,49\end{aligned}$ \\
\hline & Graminées & 18 & $\begin{array}{c}r \\
\text { Syx }\end{array}$ & & $\begin{array}{ll} & 0,821 \\
\pm & 3,44\end{array}$ & $\begin{aligned} & 0,820 \\
\pm & 3,45\end{aligned}$ & $\begin{aligned} & 0,906 \\
\pm & 2,55\end{aligned}$ \\
\hline & Total & $3 i$ & $\begin{array}{c}r \\
\text { Syx }\end{array}$ & & $\begin{array}{l}0,436 \\
\pm \quad 4,42\end{array}$ & $\begin{array}{ll} & 0,582 \\
+\quad & 4,00\end{array}$ & $\begin{aligned} & 0,827 \\
\perp & 2,80\end{aligned}$ \\
\hline \multirow{3}{*}{$\begin{array}{l}\text { Quantité de matière } \\
\text { sèche ingérée } \\
\left(g / \mathrm{kg} \mathrm{P} \mathrm{P}^{0,75}\right)\end{array}$} & Luzerne & 16 & $\begin{array}{c}r \\
\text { Syx }\end{array}$ & $\begin{aligned} & 0,409 \\
+ & 5,82\end{aligned}$ & $\begin{array}{ll} & 0,546 \\
+ & 5,33\end{array}$ & $\begin{aligned} & 0,648 \\
+\quad & 4,85\end{aligned}$ & $\begin{aligned} & 0,665 \\
+ & 4,79\end{aligned}$ \\
\hline & Graminées & 18 & $\begin{array}{c}r \\
\text { Syx }\end{array}$ & $\begin{array}{ll} & 0,876 \\
+ & 5,55\end{array}$ & $\begin{array}{ll} & 0,902 \\
\pm & 4,97\end{array}$ & $\begin{array}{l}0,891 \\
+\quad 5,22\end{array}$ & $\begin{aligned} & 0,91 \% \\
& \pm \quad 4,67\end{aligned}$ \\
\hline & Total & 34 & $\begin{array}{c}r \\
\text { Syx }\end{array}$ & $\begin{aligned} & 0,557 \\
+ & 8,18\end{aligned}$ & $\begin{aligned} & 0,860 \\
+ & 5,02\end{aligned}$ & $\begin{aligned} & 0,857 \\
+ & 5,08\end{aligned}$ & $\begin{aligned} & 0,682 \\
\pm & 7,20\end{aligned}$ \\
\hline
\end{tabular}

les fourrages verts et les ensilages, I2, 24 et 48 heures pour les foins car nous voulions pour ces derniers choisir définitivement entre les valeurs I2 et 24 heures pour la prévision de la quantité ingérée. 


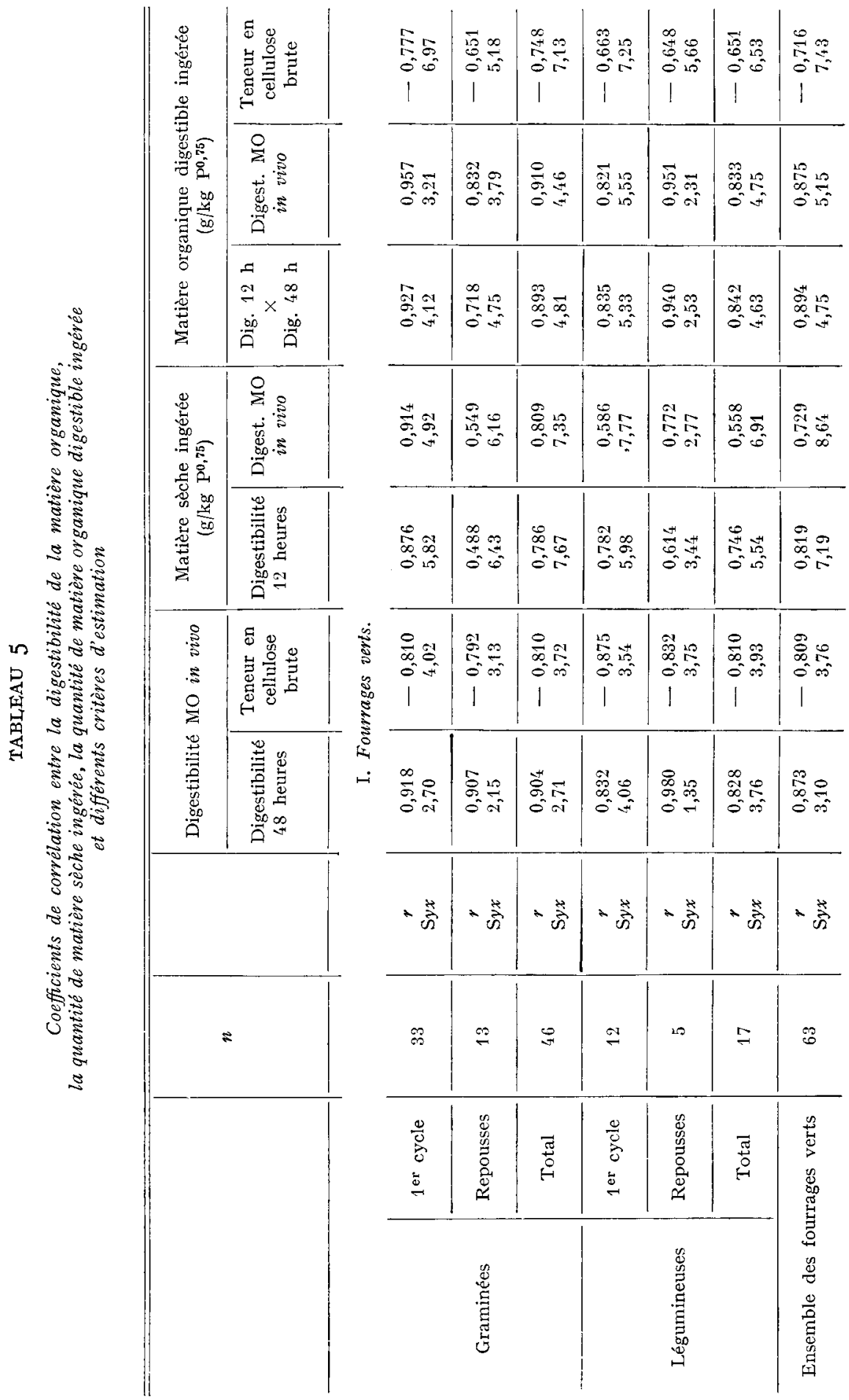




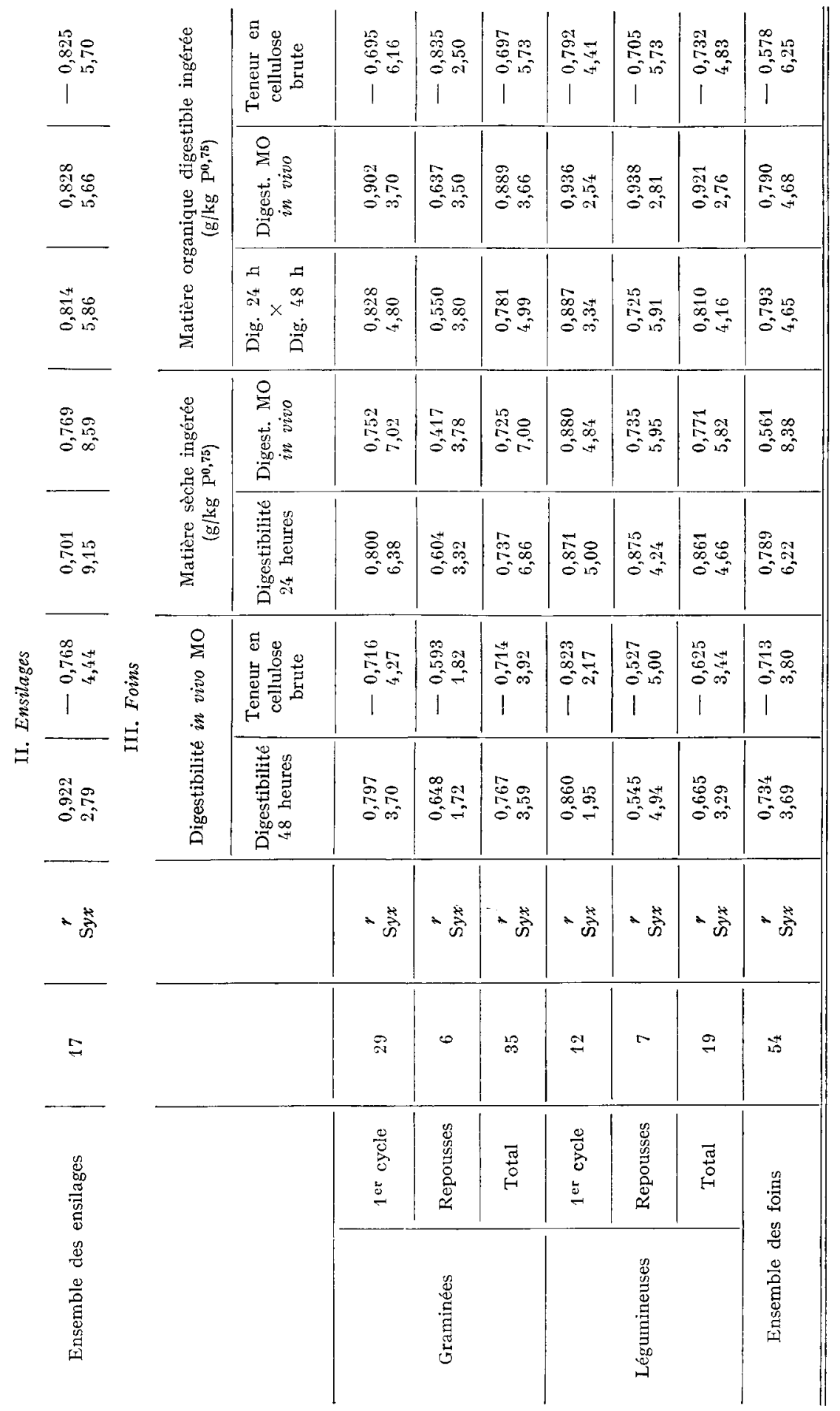


Les résultats obtenus (tabl. 5) confirment que la digestibilité in vivo peut être prévue de façon très satisfaisante à partir de la digestibilité 48 heures en sachets ; le coefficient de corrélation est égal à 0,873 pour les 63 fourrages verts, à 0,922 pour les I7 ensilages et à 0,734 pour les 54 foins. Ces valeurs sont du même ordre que celle $(0,79)$ obtenue par YANG et al. (I962) entre la digestibilité in vivo de la matière sèche et la digestibilité 44 ou 54 heures de la matière sèche en sachets de I 3 fourrages; elles auraient vraisemblablement encore été plus élevées, tout au moins pour les fourrages verts et les foins, si nous avions pu corriger les résultats đes essais I à 3 à l'aide d'un témoin comme ce fut le cas pour les ensilages. La précision de la prévision est encore améliorée si on sépare les graminées des légumineuses et les premiers cycles des repousses. Elle est supérieure à celle obtenue à partir de la digestibilité in vitro de 'TiLLEY et al. (I960) (CHENost, résultats non publiés).

La digestibilité I 2 heures en sachets permet de prévoir correctement les quantités de fourrages verts ingérés $(r=0,8 \mathrm{I} 9, n=63)$. Elle permet aussi de prévoir, mais de façon beaucoup moins précise, les quantités d'ensilage $(r=0,70 \mathrm{I})$ ou de foin $(r=0,734)$ ingérées. Ces résultats montrent d'ailleurs que la digestibilité 24 heures en sachets permet une prévision de la quantité de foin ingérée significativement meilleure $(r=0,789)$ que la digestibilité I2 heures $(r=0,734)$. Ces liaisons auraient aussi été vraisemblablement meilleures si tous les résultats avaient pu être corrigés à l'aide du même témoin.

Quoiyu'il en soit la digestibilité en sachets permet de prévoir la quantité ingérée de façon plus précise que ne le fait la digestibilité $i$ n vivo (tab1. 5).

La quantité de matière organique digestible ingérée caractérise au mieux la

TABIEAU 6

Équations de régression entre la valeur nutritive ou alimentaire des fourrages et la digestibilité en sachets 12, 24 et 48 heures

\begin{tabular}{|c|c|c|c|c|}
\hline & $\mathrm{N}$ & $y$ & $x$ & Equations de régression \\
\hline 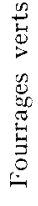 & 63 & $\begin{array}{l}\text { Digestibilité de la } \\
\text { matière organique }(\mathrm{p}, 100) \\
\text { Matière sèche ingérée } \\
\quad\left(\mathrm{g} / \mathrm{kg} \mathrm{P}^{0,75}\right) \\
\text { Matière organique diges- } \\
\text { tible ingérée }\left(g / \mathrm{kg} \mathrm{P}^{0,75}\right)\end{array}$ & $\begin{array}{l}\text { Digestibilité } 18 \text { heures } \\
\text { Digestibilité } 12 \text { heures } \\
\text { Digest. 12x digest. 48 h }\end{array}$ & $\begin{array}{l}y=0,747 x+9,35 \pm 3,10 \\
y=1,135 x+5,23 \pm 7,19 \\
y=0,879 x+3,29 \pm 4,75\end{array}$ \\
\hline 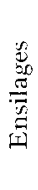 & 17 & $\begin{array}{l}\text { Digestibilité de la } \\
\text { matière organique (p. j00) } \\
\text { Matière sèche ingérée } \\
\left(\mathrm{g} / \mathrm{kg} \mathrm{P}^{0,75}\right) \\
\text { Matière organique diges- } \\
\text { tible ingérée }\left(g / \mathrm{kg} \mathrm{P}^{0,75}\right)\end{array}$ & $\begin{array}{l}\text { Digestibilité } 18 \text { heures } \\
\text { Digestibilité } 12 \text { heures } \\
\text { Digest. } 12 \times \text { digest. } 18 \mathrm{~h}\end{array}$ & $\begin{array}{l}y=0,795 x+5,02 \pm 2,79 \\
y=0,983 x-4,28 \pm 9,15 \\
y=0,785 x-4,32 \pm 5,86\end{array}$ \\
\hline$\stackrel{n}{\stackrel{n}{0}}$ & $5 / 4$ & $\begin{array}{l}\text { Digestibilité de la } \\
\text { matière organique (p. } 100) \\
\text { Matière sèche ingćrée } \\
\left(\mathrm{g} / \mathrm{kg} \mathrm{P}^{0,75}\right) \\
\text { Matière organique diges- } \\
\text { tible ingérée }\left(\mathrm{g} / \mathrm{kg} \mathrm{P}^{0,75}\right)\end{array}$ & $\begin{array}{l}\text { Digestibilité } 48 \text { heures } \\
\text { Digestibilité } 2 ' t \text { heures } \\
\text { Digest. } 2 ' x \times \text { digest. } 48 \mathrm{~h}\end{array}$ & $\begin{array}{l}y=1,038 x+9,97 \pm 3,69 \\
y=0,997 x-7,07 \pm 6,22 \\
y=0,571 x+4,61 \pm 4,65\end{array}$ \\
\hline
\end{tabular}


valeur alimentaire d'un fourrage. Elle peut être prévue de façon très satisfaisante à partir du produit digestibilité I2 heures (ou 24 heures pour les foins) $\times$ digestibilité 48 heures; le coefficient de corrélation est respectivement égal à 0,894, 0,8I4 et 0,793 pour les 63 fourrages verts, les 17 ensilages et les 54 foins. C'est à notre connaissance la meilleure relation proposée jusqu'ici; elle permet une prévision bien plus précise que la cellulose brute, du même ordre que celle obtenue à partir de la digestibilité in vivo (tab1. 5). Les équations des droites de régression liant la valeur nutritive et alimentaire des fourrages aux digestibilités en sachets sont données dans le tablea11 6 .

\section{DISCUSSION}

La technique des sachets de nylon permet de suivre la cinétique de la digestion des fourrages dans le rumen et de mettre en évidence des différences entre fourrages. Les causes des différences de vitesse de digestion doivent être recherchées dans les teneurs différentes en constituants solubles dans l'eau dont la digestion est très rapide et dans les différences de caractéristiques physico-chimiques des membranes végétales; en particulier, les luzernes sont digérées plus rapidement que les graminées, d'une part parce qu'elles sont plus riches en constituants solubles dans l'eau, d'autre part parce qu'elles sont plus pauvres en membranes digestibles qui sont par ailleurs dégradées plus rapidement que celles des graminées.

Il est normal que la digestibilité obtenue en sachets après des temps de séjour assez longs dans le rumen (égaux ou supérieurs à 48 heures) soit en liaison étroite avec la digestibilité $i n$ vivo. En effet, les constituants membranaires digestibles sont presque totalement digérés dans le rumen (90 à 95 p. Ioo d'après BRUCE et al., Ig66 et JouRneT, résultats non publiés) et leur digestion est lente. C'est d'ailleurs pourquoi le temps de séjour moyen des fourrages dans le tube digestif du mouton est pratiquement toujours supérieur à 40 heures et est le plus souvent compris entre 45 et 70 heures (DEMARQUILLY, résultats non publiés). La digestion des constituants membranaires digestibles, doit donc, pour la majorité des fourrages, être pratiquement achevée au bout de 48 heures de séjour dans le rumen. On sait en outre que les constituants solubles dans l'eau ont une digestibilité vraie voisine de roo p. roo et qu'ils sont très rapidement digérés dans le rumen. Ce qui reste dans le sachet après 48 ou 72 heures de séjour dans le rumen doit donc représenter assez fidèlement (mais un peu par excès) la fraction indigestible vraie du fourrage et par là être liée étroitement à la digestibilité in vivo. D'autres auteurs ont d'ailleurs trouvé eux aussi une relation entre la digestibilité in vivo des fourrages et la digestibilité 44 ou 54 heures de la matière sèche en sachets (YANG et al., I962) ou entre la digestibilité in vivo de la cellulose vraie et la digestibilité 42,48 ou $7^{2}$ heures de la cellulose vraie (Lusk et al., I962; Hopson et al., Ig63).

En revanche, il n'est pas étonnant que la quantité ingérée soit liée surtout à la digestibilité en sachets après un temps de séjour court dans le rumen. En effet, ce qui encombre le rumen après le repas ce n'est pas seulement la fraction indigestible (qui dépend de la digestibilité du fourrage), mais aussi la fraction digestible en cours de digestion puisque cette digestion doit avoir lieu dans le rumen, pour que la fraction indigestible soit libérée et réduite en particules suffisamment fines pour quitter le rumen. Il est donc normal que la quantité de fourrage ingérée dépende étroitement 
de la digestibilité I2 heures, c'est-à-dire non seulement de la digestibilité du fourrage, mais aussi de sa vitesse de digestion. De même, il est normal que la quantité ingérée dépende plus étroitement de la digestibilité en sachets de la matière sèche que de la digestibilité de la matière sèche non extractible à 1'eau. Ėn effet quand on relie la quantité ingérée à la digestibilité de la matière sèche non extractible à l'eau, ou à la digestibilité des constituants membranaires ou de la cellulose vraie on néglige un facteur très important de la quantité ingérée qui est la teneur en constituants solubles dans l'eau car ils sont digérés rapidement et ont un pouvoir d'encombrement très fugace. En particulier si la vitesse de digestion et par là l'acceptabilité des luzernes est supérieure à celle des graminées de même digestibilité ce n'est pas seulement parce que les membranes de luzerne sont digérées plus rapidement que celles des graminées c'est aussi et surtout parce que les luzernes sont plus riches en constituants solubles dans l'eau. Au cours d'un cycle de croissance d'une même plante, la teneur en constituants solubles dans l'eau, la digestibilité in vivo et, par là la digestibilité I2 heures, évoluent dans le même sens; tout se passe alors comme si la quantité ingérée ne dépendait que de la seule digestibilité comme l'ont proposé BLAXTER et al. (I96I). Mais il n'en est plus de même lorsqu'on considère des fourrages d'espèces ou de cycles différents. Ils pourront donc à même digestibilité être ingérés en quantités différentes s'ils sont digérés à des vitesses différentes.

L'intérêt essentiel de la méthođe des sachets est qu'elle permet suivant le temps de séjour des sachets dans le rumen, de prévoir soit la quantité ingérée soit la digestibilité, permettant done de prévoir les 2 facteurs de la valeur alimentaire des fourrages. Elle peut donc rendre de grands services, ne serait-ce qu'aux sélectionneurs qui ne disposent pas jusqu'ici de méthodes d'appréciation rapide de l'acceptabilité des fourrages. C'est ainsi que nous avons montré par exemple, que la mauvaise acceptabilité de la Fétuque élevée étudiée était due à la digestion lente de ses feuilles. Cela explique vraisemblablement pourquoi GILLET (Ig66) a observé une liaison entre la flexibilité des feuilles et 1'acceptabilité des fétuques élevées. L'inconvénient majeur de la technique des sachets, comme d'ailleurs de la technique des fermentations in vitro, est de nécessiter la présence d'animaux fistulisés. Elle se prête malheureusement moins bien au travail en série que la technique de digestibilité in vitro. Elle a cependant le mérite de rester plus proche des conditions de la digestion in vivo et, donc de permettre de mieux estimer la vitesse et l'intensité de la digestion dans le rumen. Comme toutes les méthodes biologiques elle a aussi l'inconvénient d'être moins reproductible qu'une méthode chimique. I1 sera done nécessaire d'incorporer dans chaque série des témoins et cela d'autant plus que les résultats obtenus avec des fourrages finement broyés dépendent de la qualité du tissu de nylon employé. Il est d'ailleurs vraisemblable que les liaisons que nous avons obtenues auraient été plus étroites si, dès le début, nous avions incorporé de tels témoins dans chaque série, pour corriger nos résultats.

Les inconvénients majeurs des techniques utilisant comme inoculum du jus de rumen petivent être supprimés en partie en remplaçant celui-ci par une préparation cellulasique fongique (JARRIGE, et THIVEND, I969). La digestion cellulasique permet de prévoir la digestibilité des fourrages avec une précision très satisfaisante. Il serait intéressant d'étudier si elle permet aussi de prévoir la vitesse de digestion des fourrages et par là, la quantité ingérée. 


\section{SUMMARY}

\section{STUDY OF FORAGE DIGESTION IN THE RUMEN USING SMALL NYLON BAGS RELATION TO FEED VALUE}

I. The aim of this study was to determine, if by using small nylon bags, we could observe forage digestion in the rumen. We also wished to determine if, after remaining for different lenghts of time in the rumen, the quantities of dry material digested in the bags could be related to digestibility and to the voluntary dry matter intake by sheep of the for ages.

2. 40 small nylon bags $(15 \times 7 \mathrm{~cm})$, each containing $3 \mathrm{~g}$ of ground forage, were attached to a I-kg lead ring by a short nylon string, and placed in the ventral rumen sack of a cow having a large fistula in the rumen. The bags ( 3 per forage and per length of time in the rumen) were with drawn after remaining $12,24,48$ and $7^{2}$ hours in the rumen. They were washed with water and put into a solution of pepsin acid for $4^{8}$ hours. At the end of that time, they were washed again, dried and weighed. The percentage of dry matter lost is called $12,24,48$, and 72 hours digestibility. We then studied bag digestibility of the leaves and stems of 2 lucernes and 3 grasses, cut every 2 weeks during the first vegetation cycle (4 cuttings), and those of lucerne cut at the beginning and end of the 2nd, $3^{\text {rd }}$ and $4^{\text {th }}$ vegetation cycles. Also studied were 62 whole green forage plants, $5^{8}$ hays and 17 silages, the digestibility and voluntary intake of which were measured on sheep (table I).

3. Using the same nylon bags, we followed forage digestion in the rumen (fig. I). The values obtained differ very little depending on the cow used, and the precision of estimation obtained with 3 bags was of the order of 2 p. Ioo.

4. Lucerne leaves were almost digested after 24 hours in the rumen, and the values obtained at 48 or 72 hours were about $92-93$ p. I0O, no matter what the age of the plant or the number of the vegetation cycle. On the other hand, there was considerable grass leaf lamina limb digestion between 24 and $4^{8}$ hours, and the values obtained at $4^{8}$ hours (time after which digestion was finished) decreased with the age of the plant (fig. 2 and 3 ). As was the digestion of grass stems, that of lucerne stems was slower than that of leaves, and was only finished after 48 hours in the rumen. The $4^{8}$ hour digestibility of grass stems was a ligh as that of the leaf laminas at the beginning of the first vegetation cycle, but then decreased much faster with age (fig. 2 and 3 ). The result was that 12,24 , and 48 hour digestibility of the whole plant also decreased with age (fig. 3).

5. Forages having the same in vivo digestibility had almost identical 48 or 72 -hour digestibility but very different 12 or 24 -hour digestibilities (fig. 4). Lucerne was digested faster than Italian rye-grass, and the latter faster than cocks foot or tall fescue.

6. I 2-hour digestibility depended both on the plant under consideration and 48 -hour digestibility (fig. 5). Thus, it could not alone characterize forage digestion rate. We propose to call the forage digestion rate the ratio $\frac{\mathrm{I} 2 \text {-hours digestibility }}{48 \text {-hours digestibility }} \times$ roo. Average values and extremes obtained at the beginning and end at the first vegetation cycle are presented in table 2 . They confirm that lucerne was digested more rapidly than the grasses, and Italian rye-grass more rapidly than cocks foot or tall fescue.

7. First, we tried to determine the lenght of time of digestion (1 $2,24,4^{8}$ hours) so that the digestibility and the intake of 20 green forages and 34 hays could be better predicted.

In vivo digestibility of green forages may be predicted very satisfactorily by 48 -hour digestibility $(r=0.895)$, and the dry matter intake by I 2 -hour digestibility $(r=0.9444)$ (table 3 fig. 6). In vivo hay digestibility may also be satisfactorily estimated by 48 -hour digestibiliry, an the dry matter intake by 12 or 24 -hour digestibility. (table 4 ).

8. Considering these results, we measured the bag digestibility of 42 green forages, 24 hays and 7 silages which were added to the 20 green forages and 34 hays already studied.

The results obtained (table 5) show that in vivo digestion may be predicted very satisfactorily by 48 -hour digestibility and the dry matter intake correctly predicted by 2 -hour digestibility for green forages and silages, and by 24 -hour digestibility for hay.

The intake digestible organic matter best describes the feed value of a forage. It may be predicted very satisfactorily by the product of 12-hour digestibility (or 24-hour digestibility for hays) $\times 48$-hour digestibility. The prediction is more precise than with crude cellulose and in vitro digestibility, and is of the same order as that obtained by in vivo digestibility (table 5).

These results are discussed in terms of the knowledge we have of forage chemical composition and of factors regulating the appetite in ruminants. 


\section{RÉFÉRENCES BIBLIOGRAPHIQUES}

Balch C. C., Campling R. C., I962. Regulation of voluntary food intake in ruminants. Nutr. Rev, 32, 669 .

Blaxter K. L., Wainman F. W., Wilson R. S., I96I. The regulation of food intake by sheep. Anim. Prod., 3, $5 \mathrm{I}$.

Bruce J., Goodall E. D., Kay R. N. B., Phillipson A. T., Vowles L. E., Ig66. The flow of organic and inorganic materials through the alimentary tract of the sheep. Proc. Royal Soc., série B, 166, 46-63.

Corbett J. L., Langlands J. P., Reid G. W., Ig63. Effects of season of growth and digestibility of herbage on intake by grazing dairy cows. Anim. Prod., 5, II9-129.

Crampton E. W., I957. Interrelations between digestible nutrient and energy content, voluntary dry matter intake, and the overall feeding value of forages. J. Animal Sci., 16, 546-552.

Demarquiliy C., 1965. Factors affecting the voluntary intake of green forage by sheep. Proc. 9th Intern. Grassland Congress. Sao-Paulo, Section 9 ; o. p. 400 pp. 877-885.

Demarovilly C., Jarrige R., I964. Valeur alimentaire de l'herbe des prairies temporaires aux stades d'exploitation pour le pâturage. I. Composition chimique et digestibilité. Ann. Zootech., 13, 301-339.

Demarguiley C., 1966. Valeur alimentaire de l'herbe des prairies temporaires aux stades d'exploitation pour le pâturage. II. Quantité ingérée par les vaches laitières. Ann. Zootech., 15, I47-I69.

Donefer E., Crampton E. W., Lloyd L. L., ig6o. Prediction of the nutritive value index of a forage from in vitro rumen fermentation data. J. Animal Sci., 19, 545-55I.

Gillet M., JAdAs-Hecart J., I965. Leaf flexibility, a character for selection of tall fescue for palatability. Proc. 8th Intern. Grassland Congress. Sao-Paulo, 1, o. p. 595 Pp. I 55.

Hopson D. J., Johnson R. R., Dehority B. A., 1963. Evaluation of the dacron bag technique as a method for measuring cellulose digestibility and rate of forage digestion. J. Animal Sci., 22, 448-453.

Ivins J. D., I955. The palatability of herbage. Herbage Abstr., 25, 75-79.

Jarrige R., Thivend P., I969. Action d'une cellulase fongique sur les membranes et son intérêt pour prévoir la digestibilité des plantes fourragères. Ann. Biol. anim. Bioch. Biophys., 9, I7 I-Igo.

Johnson R. R., I966. Techniques and procedures of in vitro and in vivo rumen studies. J. Animal Sci. $\mathbf{8}, 855-875$.

Journet M., I965. Résultats non publiés.

Lusk J. W., Browning C. B., Miles 'T. T., I962. Small sample in vivo cellulose digestion procedure for forage evaluation. J. Dairy Sci., 45, 69-73.

Quin J. I., Van der Wath J. C., Mүвrugh S., 1938. Studies of the alimentary tract of Merino sheep in South Africa. IV. Description of experimental technique. Onderstepoort J. Vet. Sci. Animal Ind., 11, 34I.

Tilley J. M. A., Deriaz R. E., TerRy R. A., I960. The in vitro measurement of herbage digestibility and assessment of nutritive value. Proc. 8th Grassland Congress, Reading, 533-537.

Tilley J. M. A., Terry R. A., 1963. A two stage technique for the in vitro digestion of forage crops. J. Brit. Grassland Soc., 18, I04-III,

Van Keuren R. W., Heinemann W. W., ig62. Study of a nylon bag technique for in vivo estimation of forage digestibility. J. Animal Sci., 21, 340-345.

Yang M. G., Ingalls J. R., Thomas J. W., I962. Dry matter disappearance in nylon bag suspended in rumen as affected by heat, time and rations and its use in forage evaluation. J. Dairy $5 c i ., 45,676$. 